\title{
La prison chevillée au corps. Pour une approche géographique du placement sous surveillance électronique
}

\section{Franck Ollivon}

\section{(2) OpenEdition}

Journals

Édition électronique

URL : https://journals.openedition.org/cdg/7473

DOI : $10.4000 / \mathrm{cdg} .7473$

ISSN : 2107-7266

Éditeur

UMR 245 - CESSMA

Référence électronique

Franck Ollivon, « La prison chevillée au corps. Pour une approche géographique du placement sous surveillance électronique », Carnets de géographes [En ligne], 15 | 2021, mis en ligne le 30 avril 2021, consulté le 28 mai 2021. URL : http://journals.openedition.org/cdg/7473 ; DOI : https://doi.org/ $10.4000 / \mathrm{cdg} .7473$

Ce document a été généré automatiquement le 28 mai 2021.

\section{(†) $\ominus$}

La revue Carnets de géographes est mise à disposition selon les termes de la Licence Creative Commons Attribution - Pas d'Utilisation Commerciale - Pas de Modification 4.0 International. 


\title{
La prison chevillée au corps. Pour une approche géographique $\mathrm{du}$ placement sous surveillance électronique
}

\author{
Franck Ollivon
}

1 Mes recherches doctorales partent du constat que, si la prison focalise encore l'attention du plus grand nombre, elle ne représente pourtant qu'une mesure pénale parmi d'autres. Au cours du second $\mathrm{xx}^{\mathrm{e}}$ siècle sont ainsi apparues des alternatives à l'incarcération de natures très diverses: travail d'intérêt général, sursis-mise à l'épreuve, libération conditionnelle... Dès lors, en France comme dans de nombreux pays occidentaux, un rapide aperçu des statistiques pénitentiaires montre que la peine de prison est largement concurrencée par ces mesures alternatives dites «en milieu ouvert » parce qu'elles se déroulent au moins en partie en-dehors d'un établissement pénitentiaire. Ainsi, en France, au $1^{\mathrm{er}}$ janvier 2019, un peu plus de 70000 personnes étaient détenues en milieu fermé contre près de 170000 suivies en milieu ouvert.

2 Cette évolution de la justice pénale reste largement ignorée des sciences sociales en général et de la géographie en particulier. Dans le prolongement de la legal geography (Blomley et al., 2001), certains travaux récents ont certes fait valoir les contradictions entre le principe constitutionnel d'égalité des citoyens sur le territoire national et les variations territoriales dans l'exercice concret de la justice pénale (Cahu, 2017). Ces réflexions portent toutefois sur l'amont de la peine, sur le temps du procès pénal, et délaissent cette dimension essentielle de l'activité judiciaire qu'est l'exécution de la peine. Celle-ci reste en fait principalement travaillée par une géographie dite « carcérale » (Moran, 2015) qui a souligné les limites de la notion d'« institution totale » empruntée à Erving Goffman (Bony, 2015), sans pour autant pleinement interroger ce mouvement de « détotalisation » de l'institution que constitue l'essor du milieu ouvert ${ }^{1}$. Ma thèse porte plus spécifiquement sur l'une de ces mesures, sans doute la plus médiatique, le placement sous surveillance électronique (PSE) communément appelé 
«bracelet électronique ». Le PSE est en fait une forme d'assignation à résidence : le placé a obligation de se trouver à son domicile sur certaines plages horaires définies par un magistrat, ce que contrôle un boîtier installé à son domicile qui mesure par fréquence radio la présence d'un émetteur fixé à sa cheville.

Dès lors, le PSE pose un problème majeur au géographe. Vanté comme le meilleur remède aux maux de la prison (coût, surpopulation et récidive), le PSE est une peine qui appartient au milieu ouvert dans la mesure où il se déroule hors de tout établissement pénitentiaire, dans des lieux qui peuvent être qualifiés « d'ordinaires » par rapport au régime d'exception qui accompagne l'incarcération. Mais dans le même temps, le PSE conserve la trace du milieu fermé dans la mesure où l'espace de la peine reste fragmenté par les impératifs horaires qui s'appliquent au placé. Le PSE constitue donc une mesure hybride qui interroge les territorialités pénales contemporaines, c'est-àdire les stratégies spatiales par lesquelles l'institution judiciaire prétend punir et réinsérer le condamné. Le PSE est-il toujours une " peine géographique » au sens d'une peine qui « punit par l'espace » (Milhaud, 2015) ? Autrement dit, dans une alternative à l'incarcération telle que le PSE, que reste-t-il de la contrainte spatiale inhérente au modèle carcéral ? Une technologie de surveillance peut-elle se substituer aux murs et aux grilles de la prison? La nature de ces questions situe la thèse au croisement entre surveillance studies (Lyon, 1994), legal geography (Blomley et al., 2001) et carceral geography (Moran, 2015).

4 Mon travail empirique associe dès lors trois méthodes distinctes. Il repose d'abord sur une démarche ethnographique d'observation qui doit permettre de saisir en actes les pratiques des agents des services pénitentiaires d'insertion et de probation (SPIP) chargés de réaliser la surveillance et l'accompagnement social des placés. Je mobilise ensuite des entretiens semi-directifs avec des acteurs institutionnels - conseillers pénitentiaires d'insertion et de probation (CPIP) et magistrats principalement - et une trentaine de placés rencontrés pendant mes journées d'observation. Enfin, je m'appuie sur une enquête par questionnaires qui a pour principale fonction de combler une absence totale d'informations statistiques sur les placés et les lieux dans lesquels ils se trouvent assignés à résidence. Ma recherche porte sur deux départements rhônealpins, la Haute-Savoie et le Rhône. Il s'agit ainsi de varier les terrains afin de mieux saisir les « modalités d'actualisation locale du droit » (Melé, 2009).

5 La thèse aboutit à trois conclusions principales. Elle montre tout d'abord qu'avec le PSE comme avec la prison, l'espace reste une dimension fondamentale du dispositif pénal. Il est ce par quoi l'institution prétend réinsérer puisque le PSE s'adosse à ce spatialisme qui superpose insertion sociale et insertion spatiale. Toutefois, l'espace porte aussi la dimension « rétributive » de la peine dans la mesure où le placé est confiné à un espace certes ordinaire mais défini et construit par l'institution. En d'autres termes, avec le PSE, les lieux de résidence, de travail, de soin, de loisirs, tous ces lieux du quotidien dont l'accès est réglementé par l'institution judiciaire, participent aussi bien de la contrainte - et donc de la sanction - que de la liberté.

6 La thèse souligne ensuite la complexité de la fabrique du territoire de la peine en milieu ouvert. Les discontinuités qui s'imposent aux condamnés sont déterminées au cas par cas par les fonctionnaires du ministère de la Justice, en suivant des critères juridiques et techniques mais aussi au nom d'imaginaires spatiaux individuels. Néanmoins, en renvoyant la peine au milieu ouvert, le PSE s'inscrit dans un espace anisotrope : les logements - et donc les lieux d'assignation - ne sont pas les mêmes, les localisations 
résidentielles - et donc les temps de sortie - ne sont pas les mêmes. Dès lors, le PSE est une peine socialement sélective qui exclut les condamnés précaires ou du moins les contraint à d'instables arrangements avec un tiers, qu'il soit celui qui héberge ou celui qui emploie. Ce tiers acquiert sur eux, du fait même de la peine, un certain pouvoir : perdre un logement ou perdre un emploi peut signifier, pour le placé, l'incarcération ou la réincarcération. Plus encore, par rapport au milieu fermé que réglementent les législations française et européenne, les conditions matérielles dans lesquelles se déroule le PSE restent peu codifiées: de la chambre de foyer jusqu'à la villa de trois étages avec jardin, tout logement peut accueillir un PSE. Cette mesure pénale réinjecte donc au cœur de la peine des inégalités sociales qui certes lui préexistent mais prennent, en raison même de la peine, une importance toute particulière pour les placés.

Enfin, au-delà de la diversité des conditions matérielles de la peine, la thèse interroge la sensation d'enfermement que décrivent les placés en entretien. L'appareillage technique sur lequel repose la surveillance constitue certes l'une des dimensions explicatives de cette sensation. Toutefois, le facteur technique ne suffit pas à la comprendre puisque les placés font peu l'expérience de ce contrôle technologiquement médié, qui connaît en outre de multiples défaillances ou imprécisions, humaines comme techniques. Dès lors, ma fréquentation des SPIP et des placés sous surveillance électronique m'a donné à penser l'importance des discours juridiques et techniques qui accompagnent le PSE et interviennent directement dans la production des discontinuités spatiales inhérentes à cette peine. Certains actes procéduraux rituels viennent soutenir cette performativité des discours judiciaires qui assimilent l'espace d'assignation à la prison. Plus qu'un simple état administratif ou une situation matérielle, l'enfermement est donc une sensation que les discours des agents de l'institution, de leurs relais et des placés eux-mêmes participent à produire.

\section{Discipline \\ Géographie \\ Directrice \\ Isabelle Lefort \\ Université \\ Université Lumière Lyon 2}

Membres du jury de thèse, soutenue le 30/11/2018

Marie-Sophie Devresse, Professeure en criminologie, Université Catholique de Louvain (rapporteure)

Isabelle Lefort, Professeure en géographie, Université Lumière Lyon 2 (directrice) Michel Lussault, Professeur en géographie, Ecole Normale Supérieure de Lyon Olivier Milhaud, Maître de conférences en géographie, Université Paris Sorbonne Marie Morelle, Maîtresse de conférences HDR en géographie, Université Paris 1 Panthéon-Sorbonne (rapporteure)

\section{Situation professionnelle à l'issue de la thèse}

Attaché temporaire d'enseignement et de recherche à l'Ecole Normale Supérieure 


\section{BIBLIOGRAPHIE}

BLOMLEY N., DELANEY D., FORD R. T. (2001), The legal geographies reader : law, power, and space, Oxford, Blackwell.

BONY L. (2015), « La prison, une "cité avec des barreaux" ? Continuum socio-spatial par-delà les murs », Annales de géographie, no. 702-703, pp. 275-299.

CAHU É. (2017), Géographie de la justice pénale en France. L'équité à l'épreuve des territoires, Thèse de doctorat, Normandie Université.

GOFFMAN E. (1961), Asylums: Essays on the Social Situation of Mental Patients and Other Inmates, New York, Anchor Books.

LYON D. (1994), The Electronic Eye: The Rise of Surveillance Society, Minneapolis, University of Minnesota Press.

MELÉ P. (2009), « Pour une géographie du droit en action », Géographie et cultures, no. 72, pp. $25-42$.

MILHAUD O. (2015), «L'enfermement ou la tentation spatialiste. De "l'action aveugle, mais sûre" des murs des prisons ", Annales de géographie, no. 702-703, pp. 140-162.

MORAN D. (2015), Carceral Geography: Spaces and Practices of Incarceration, Farnham, Routledge.

\section{NOTES}

1. Erving Goffman définit l'" institution totale » comme " a place of residence and work where a large number of like-situated individuals, cut off from the wider society for an appreciable period of time, together lead an enclosed, formally administered round of life » (1961, p. xiii). La notion a pour ambition de designer une grande variété de lieux de réclusion dont l'une des principales caractéristiques tient à la coupure qu'ils produisent avec l'extérieur.

\section{INDEX}

Thèmes : Carnets de soutenances 


\section{AUTEUR}

\section{FRANCK OLLIVON}

Attaché temporaire d'enseignement et de recherche à l'Ecole Normale Supérieure.

franck.ollivon[at]ens.psl.eu 